\title{
Why topology in the minimalist foundation must be pointfree
}

\author{
Maria Emilia Maietti and Giovanni Sambin \\ Dipartimento di Matematica, Università di Padova \\ via Trieste 63, 35121 Padova (Italy) \\ \{maietti, sambin\}@math.unipd.it
}

\begin{abstract}
We give arguments explaining why, when adopting a minimalist approach to constructive mathematics as that formalized in our two-level minimalist foundation, the choice for a pointfree approach to topology is not just a matter of convenience or mathematical elegance, but becomes compulsory. The main reason is that in our foundation real numbers, either as Dedekind cuts or as Cauchy sequences, do not form a set.
\end{abstract}

MSC 2000: 03G30 03B15 18C50 03B20 03F55

Keywords: pointfree topology, real numbers, choice sequences, Bar Induction, constructive type theory, axiom of unique choice, minimalist foundation.

\section{Introduction}

Beginning with [MS05], we embarked in the project of building a minimalist foundation to develop constructive mathematics. By constructive mathematics we here mean one developed in an intuitionistic and predicative foundation. There are however different constructive foundations as well as different philosophical attitudes to constructivism (see for example the preface of [Tv88]).

We decided to build a minimalist foundation, such that mathematics developed in it is compatible with the different approaches to constructivism, and also with classical mathematics. A full formal system, called "minimalist foundation", was proposed in [Mai09]. In parallel, also a new approach to constructivism, called "minimalist", was put forward in [Sam08, Sam11, Sam12]. This is inspired by the constructive approach originated explicitly with Brouwer in the beginning of last century and revived in the 60s and 70s by Bishop [Bis67], Martin-Löf [Mar70] and others.

The main purpose of the present paper is to give arguments showing that, adopting our minimalist foundation, the predicative pointfree approach of formal topology, introduced by Martin-Löf and the second author in the 80s [Sam87], is compulsory and not just an option (a matter of convenience, mathematical elegance,...).

Constructive pointfree topology constitutes an alternative (see for example [Pal05b]) to point-wise approaches to constructive analysis by Brouwer (see [Tv88]) and Bishop (see [Bis67, BB85]). The advantage of the pointfree approach is that of being in accordance with a computational view of mathematics, as exemplified by the Kleene realizability interpretation of logic and Heyting arithmetic and advocated by Martin-Löf in [Mar70].

A priori, using Martin-Löf's type theory in [NPS90] as a foundation, also Bishop's approach could seem legitimate. In fact, one can define a point-wise topology on Dedekind real numbers, because these are in bijective correspondence with Cauchy sequences and the latter can be represented in Martin-Löf's type theory as a quotient set, formally a setoid, that is a set together with an equivalence relation. Also in the predicative foundation of Aczel's Constructive set theory CZF [Acz78, Acz82, Acz86] both Dedekind reals and Cauchy reals form a set and a point-wise approach is also possible.

According to our minimalist attitude, this should not be the case if we want to work in a constructive foundation compatible with classical predicativity. As we expected, in [Mai12] it is indeed shown that in 
our minimalist foundation real numbers, either following Dedekind (Dedekind cuts) or Cantor (Cauchy sequences), do not form a set, but only a proper collection. Also choice sequences of Baire and Cantor spaces do not form a set.

The main reason is due to the fact that in our foundation functions do not generally form a set. We obtain a set by restricting to operations, that is to a type-theoretic notion of function. The non derivability of choice principles, including unique choice which identifies a functional relation with an operation, makes operations and functions two distinct concepts. Such concepts are then used to define lawlike sequences on natural numbers as operations, and choice sequences as functions.

The fact that real numbers or choice sequences do not form a set in our minimalist foundation does not forbid developing constructive analysis. In fact, we can introduce effectively, via an inductive definition, a formal topology characterizing the pointfree structure over real numbers, or over choice sequences in Baire and Cantor spaces. Then real numbers or choice sequences are themselves defined as formal points of such formal topologies. They are ideal entities (see [Sam12]) in the sense that they form a proper collection.

All this leads us to conclude that if we want to develop constructive topology, including that of real numbers, in our minimalist foundation, we must follow the pointfree approach, through which to distinguish the real (effective) structure of a topology from a corresponding ideal (infinitary) structure of formal points.

An important advantage of developing topology in such a predicative way is that it becomes compatible with the most relevant foundations, from the predicative constructive ones as Martin-Löf's type theory and Aczel's CZF, to the internal theory of topoi and classical predicative systems as Feferman's theories in [Fef79]. Our foundation is compatible with such different systems because it contains two different levels of abstraction. We got to the idea of a two-level system in [MS05], very briefly for the following reason. We realized that we wished to retain the best aspects of available constructive foundations: on one hand, we wished to formulate it in an extensional language close to the language of present day mathematics (as Aczel's CZF), on the other hand we wanted to formulate it as an intensional type theory (as Martin-Löf's intensional one) to make its computational contents explicit via the extraction of programs from proofs. In [MS05] we proved that this combination was possible only by building a two-level foundation, with an intensional level and an extensional one linked by an interpretation that restores the computational information needed at the intensional level to represents the extensional constructions, according to the forget-restore principle proposed by the second author in [SV98]. The two-level minimalist foundation in [Mai09] fulfils these requirements and we can compare it with other theories at the appropriate level: we can compare its intensional level with intensional theories as Martin-Löf's one, and its extensional level with extensional theories as Aczel's CZF and other axiomatic set theories.

Lastly, the intensional level could serve as a base for a minimalist proof-assistant whose formalized proofs can, a priori, be reused in proof-assistants based on the many extensions. The minimalist foundation can then well serve as a basic theory where to compare the different approaches to topology and their proofs.

\section{The need of a minimalist foundation.}

A plurality of philosophical reasons for a constructive approach to mathematics have been proposed, both before and after Brouwer around a century ago.

Presently, various logical systems to formalize constructive mathematics are available in the literature. They range from axiomatic set theories, as Aczel's CZF [Acz78, Acz82, Acz86] or Friedman's IZF [Bee85], to the internal set theory of categorical universes as topoi or pretopoi [MM92, JM95, Mai05b], to type theories as Martin-Löf's type theory [NPS90] or Coquand's Calculus of Inductive Constructions [Coq90, CP90]. No existing constructive foundation has yet superseded the others as the standard one, as Zermelo-Fraenkel axiomatic set theory did for classical mathematics.

Also various machine-aided proof development systems are available to implement mathematics (see, for example, [Wie06]). Many of those for constructive mathematics are based on type systems which are also paradigm of (functional) programming languages with the possibility of extracting the com- 
putational contents of constructive mathematical proofs. Some of these, as for example Coq [Coq10] or Matita [ARCT11], are based on impredicative typed systems, while some other, as for example Agda [BDN09] and Nuprl $\left[\mathrm{ABC}^{+} 06\right]$, are based on predicative ones.

Beginning with [MS05], we embarked in the project of developing a foundation with a minimal number of assumptions. The main reason for this choice is to support our general attitude to preserve all effective notions and conceptual distinctions as much as possible, with no a priori exception. The result is a foundation which is minimalist also in the sense that it becomes the common core among the most relevant constructive foundations. Thus we expect that such a minimalist foundation should be useful not only for constructive mathematicians but also to logicians, for example as a base system to do constructive reverse mathematics, and also to computer scientists, as a base for a minimalist proof-assistant suitable for formalizing reusable proofs and program extraction from proofs.

\subsection{The need of a two-level theory}

In our opinion, a constructive foundation should make evident those key aspects which differentiate constructive mathematics from classical mathematics. For example, a typical characteristic of constructive proofs, contrary to classical ones, is the possibility of extracting programs computing witnesses of true existential statements occurring in them.

Even better, any proof in a constructive system should be seen as a program. Hence, a foundation for constructive mathematics should be at the same time a theory of sets, in which to formalize mathematical theorems, and a programming language, in which to extract the computational contents of mathematical proofs.

In [MS05] we argued that such a constructive foundation should be a two-level theory. One level, called extensional, should be an extensional set theory (with undecidable equality of sets and elements) formulated in a language close to that used in the common practice of developing mathematics. Another level, called intensional, should be an intensional theory (with decidable equality of sets and elements) enjoying extraction of programs from proofs. ${ }^{1}$ Then, in order to guarantee the extraction of programs even from proofs written at the extensional level, we required that the extensional level should be obtained as an abstraction of the intensional level according to the forget-restore principle proposed by the second author in [SV98].

The link between the two levels was then made more technical in [Mai09], by requiring that the extensional level should be interpreted in the intensional one by means of a quotient completion of the latter, i.e. to see the extensional level as (a fragment of) the internal language of a quotient completion built on the intensional one.

This kind of link captures what happens in the practice of computer-aided formalization of mathematics in an intensional type theory, which makes use of the so called model of "setoids" built on it (see [Hof97, BCP03]). Actually another motivation behind the notion of two-level foundation in [MS05, Mai09] is the desire of making explicit the extensional theory validated in the quotient model used to formalize mathematical proofs in intensional type theory.

\section{The minimalist foundation}

In [Mai09] we presented a two-level formal system which satisfies the requirements in [MS05] of a twolevel foundation for constructive mathematics. We call this system the two-level minimalist foundation. We are aware, however, that a specific formal system, which is static by definition, cannot fully capture the dynamics of the minimalist approach to constructivism, started in [MS05, Sam08, Sam11, Sam12].

The two levels of the minimalist foundation are both given by a type theory à la Martin-Löf: the intensional level, called mTT, is an intensional type theory including aspects of Martin-Löf's one in [NPS90] (and extending the set-theoretic version in [MS05] with collections), and its extensional level, called emTT, is an extensional type theory including aspects of extensional Martin-Löf's one in [Mar84]. Then a quotient model of setoid à la Bishop [Bis67, Hof97, BCP03, Pal05a] over the intensional level

\footnotetext{
${ }^{1}$ Actually in [MS05] we required the intensional theory to be a proofs-as-programs theory, i.e. a theory consistent with the axiom of choice and the formal Church thesis.
} 
is used in [Mai09] to interpret the extensional level in the intensional one. A categorical study of this quotient model has been carried on in [MR13, MRara, MRarb] and related to the construction of Hyland's effective topos [Hyl82, HJP80].

In the following we explain the main characteristics of the extensional level emTT viewed more as a many sorted logic than as a type theory. This is because both levels of the minimalist foundation are given by a type theory that includes a primitive notion of proposition, which allows us to control the validity of choice principles.

Need of two types of entities: sets and collections. A minimalist foundation for constructive mathematics should certainly be based on intuitionistic predicate logic and include at least the axioms of Heyting arithmetic. Hence we could expect to build it starting from a many-sorted logic, such as Heyting arithmetic of finite types [Tv88], where sorts, that we call types, include the basic sets we need to represent our mathematical entities.

However, in order to develop topology in an intuitionistic and predicative way, we need a foundation with two kinds of entities: sets and collections. The main reason is that the power of a non-empty set, namely the discrete topology over a non-empty set, fails to be a set in a predicative foundation, and it is only a collection.

Need of two types of propositions. In parallel with the presence of sets and collections, to keep the system predicative we also need to distinguish two types of propositions: those closed under quantifications on sets, called here small propositions as in [Mai09] (and proper propositions in [Sam14]), from those closed under any kind of quantification, called here simply propositions as in [Mai09] (and improper propositions in [Sam14]).

Need of two types of functions. It is well known that adding the principle of excluded middle to some constructive foundations, as Aczel's CZF or Martin-Löf's type theory, one can derive that powercollections become sets and thus get an impredicative theory. In both such theories this is due to the fact that the collection of functions from a set $A$ to the boolean set $\{0,1\}$, called exponentiation of the boolean set over $A$, forms a set, too. Therefore, if we wish compatibility with classical theories where the power of a non-empty set is not a set as in Feferman's predicative theories [Fef79], we need to avoid exponentiation of functions.

A drastic solution is to drop all axioms yielding any form of exponentiation. What we propose is to allow exponentiation only of a certain kind, as it happens in [Fef79]. To this purpose, we introduce a primitive notion of operation, represented by certain functional terms

$$
f(x) \in B[x \in A]
$$

in a set $B$ with a free variable in the set $A$. These operations can be defined as type-theoretic functions of a type theory, like in Martin-Löf's type theories [NPS90, Mar84]. Clearly any operation $f(x) \in B[x \in A]$ must give rise to a functional relation $f(x)={ }_{B} y[x \in A, y \in B]$, i.e. what is usually called function. What we do not wish to guarantee is the converse. Our idea is then that only exponentiation of operations from a set $A$ to a set $B$ forms a set.

\subsection{The main types of the minimalist foundation}

The formal system emTT of the extensional level of the minimalist foundation in [Mai09] is written in the style of Martin-Löf's type theory [NPS90] by means of the following four kinds of judgements:

$$
\text { A type }[\Gamma] \quad A=B \text { type }[\Gamma] \quad a \in A[\Gamma] \quad a=b \in A[\Gamma]
$$

that is the type judgement (expressing that something is a specific type), the type equality judgement (expressing when two types are equal), the term judgement (expressing that something is a term of a certain type) and the term equality judgement (expressing the definitional equality between terms of the same type), respectively, all under a context $\Gamma$. 
The word type is used as a meta-variable to indicate four kinds of entities: collections, sets, propositions and small propositions, namely

$$
\text { type } \in\left\{\text { coll, set, prop, } \text { prop }_{s}\right\}
$$

Therefore, in emTT types are actually formed by using the following judgements:

$$
A \text { set }[\Gamma] \quad B \text { coll }[\Gamma] \quad \phi \operatorname{prop}[\Gamma] \quad \psi \operatorname{prop}_{s}[\Gamma]
$$

saying that $A$ is a set, that $B$ is a collection, that $\phi$ is a proposition and that $\psi$ is a small proposition.

Here, contrary to [Mai09] where we use only capital latin letters as meta-variables for all types, we use greek letters $\psi, \phi$ as meta-variables for propositions and capital latin letters $A, B$ as meta-variables for set or collections, and small latin letters $a, b, c$ as meta-variables for terms, i.e. elements of the various types.

Observe that for a set $A$, when we say that

$$
a \in A[\Gamma]
$$

is derivable in emTT, we actually mean that the term $a$ is an element of the set $A$ under the context $\Gamma$ and hence the symbol $\in$ stands for a set membership. As usual in type theory, equality of sets is given primitively and is not defined by equating sets with the same elements. This is indeed a main difference between a set theory defined as a typed system in the style of Martin-Löf's type theory [NPS90] with respect to an axiomatic set theory à la Zermelo-Fraenkel.

We now proceed by briefly describing the various kinds of types in emTT, starting from small propositions and propositions, then sets and finally collections.

Small propositions in emTT include all the logical constructors of intuitionistic predicate logic with equality and quantifications restricted to sets:

$$
\phi \operatorname{prop}_{s} \equiv \perp|\phi \wedge \psi| \phi \vee \psi|\phi \rightarrow \psi| \forall x \in A \phi(x)|\exists x \in A \phi(x)| x=_{A} y
$$

provided that $A$ is a set. Here we use the more familiar $x={ }_{A} y$ for the extensional equality type $\mathrm{Eq}(A, a, b)$ of Martin-Löf type theory in [Mar84].

Then, propositions of emTT include all the logical constructors of intuitionistic predicate logic with equality and quantifications on all kinds of types, i.e. sets and collections. Of course, small propositions are also propositions.

$$
\phi \text { prop } \equiv \phi \text { prop }_{s}|\phi \wedge \psi| \phi \vee \psi|\phi \rightarrow \psi| \forall x \in B \phi(x)|\exists x \in B \phi(x)| x={ }_{B} y
$$

In order to close sets under comprehension, for example to include the set of positive natural numbers $\{x \in \mathrm{N} \mid x \geq 1\}$, and to define operations on such sets, we need to think of propositions as types of their proofs: small propositions are seen as sets of their proofs while generic propositions are seen as collections of their proofs. That is, we add to emTT the following rules

$$
\left.\operatorname{prop}_{s} \text {-into-set) } \quad \frac{\phi \text { prop }_{s}}{\phi \text { set }} \quad \text { prop-into-coll }\right) \quad \frac{\phi \text { prop }}{\phi \text { coll }}
$$

The difference between the notion of set and collection will be explained later.

A key feature of the extensional typed system emTT is proof irrelevance of propositions. This means that in emTT a proof of a proposition, if it exists, is unique and equal to a canonical proof term called true thanks to the following rules

$$
\text { prop-mono) } \frac{\phi \text { prop }[\Gamma] \quad p \in \phi[\Gamma] q \in \phi[\Gamma]}{p=q \in \phi[\Gamma]} \quad \text { prop-true) } \frac{\phi \text { prop } \quad p \in \phi}{\text { true } \in \phi}
$$

Proof-irrelevance of propositions justifies the introduction of a judgement asserting that a proposition $\phi$ is true under a context $\Gamma$ and supposing propositions $\psi_{1}, \ldots, \psi_{m}$ true as in [Mar84, Mar85]. This judgement can be directly interpreted in emTT as follows:

$$
\phi \text { true }\left[\Gamma ; \psi_{1} \text { true }, \ldots, \psi_{m} \text { true }\right] \equiv \operatorname{true} \in \phi\left[\Gamma, y_{1} \in \psi_{1}, \ldots, y_{m} \in \psi_{m}\right]
$$


In emTT sets are characterized as inductively generated types and they include the following:

$$
\text { A set } \equiv \phi \operatorname{prop}_{s}\left|N_{0}\right| N_{1}|\operatorname{List}(A)| \Sigma_{x \in A} B(x)|A+B| \Pi_{x \in A} B(x) \mid A / \rho
$$

where the notation $N_{0}$ stands for the empty set, $N_{1}$ for the singleton set, List $(A)$ for the set of lists on the set $A, \Sigma_{x \in A} B(x)$ for the indexed sum of the family of sets $B(x)$ set $[x \in A]$ indexed on the set $A$, $A+B$ for the disjoint sum of the set $A$ with the set $B, \Pi_{x \in A} B(x)$ for the product type of the family of sets $B(x)$ set $[x \in A]$ indexed on the set $A$, and $A / \rho$ for the quotient set provided that $\rho$ is a small equivalence relation $\rho$ prop $_{s}[x \in A, y \in A]$. Moreover, we call $\mathrm{N}$ the set of natural numbers represented by $\operatorname{List}\left(N_{1}\right)$.

The notion of set in emTT agrees with that in Bishop [Bis67] and Martin-Löf [Mar70]. According to them sets must have an effective nature which is mostly forgotten in any axiomatic approach, when a universe of sets closed under certain properties is assumed as given. In fact, each set $A$ must be specified by providing a finite number of rules to construct all its elements (see the rules of emTT forming elements of sets in [Mai09]). It is understood that the rules defining a set are inductive, that is, their application can be iterated any finite number of times. The infinite is only potential, and in a certain sense it is always reduced to a finite description, at a higher order: not a finite number of elements, but a finite number of rules to generate (the infinite number of) them. In particular the elements of the product type $\Pi_{x \in A} B(x)$ are only terms

$$
b(x) \in B(x)[x \in A] .
$$

In the case the family $B(x)$ set $[x \in A]$ is just a constant set $B$ indexed on the set $A$, then we indicate the product type simply as

$$
A \rightarrow B \equiv \Pi_{x \in A} B
$$

and its elements are just operations

$$
b(x) \in B[x \in A]
$$

Hence, in emTT operations between two sets form a set, but generic functions between them do not.

Finally, collections in emTT include the following types: ${ }^{2}$

$$
B \text { coll } \equiv A \text { set } \mid \phi \text { prop }|\mathcal{P}(1)| A \rightarrow \mathcal{P}(1) \mid \Sigma_{x \in B} \phi
$$

where $\mathcal{P}(1)$ and $A \rightarrow \mathcal{P}(1)$ stand for the power-collections of the singleton and of a set $A$ respectively, and $\Sigma_{x \in B} \phi$ stands for the indexed sum of the propositional function $\phi$ prop $[x \in B]$ indexed on the collection $B$. Actually, for a set $A$, we will use the common abbreviation of power-collection

$$
\mathcal{P}(A) \equiv A \rightarrow \mathcal{P}(1)^{3}
$$

Elements of the power-collections rely on the notion of subset, which in emTT is inspired by that in [SV98] put on top of Martin-Löf's type theory. A subset of a set $A$ is defined as the equivalence class of a small propositional function $\phi(x)$ depending on one argument in $A$ with respect to the equivalence relation of equiprovability. This is the minimum we must require in order to close subsets under comprehension. Indeed, for any small propositional function $\phi(x) \operatorname{prop}_{s}[x \in A]$ on a set $A$ we can define its subset comprehension as

$$
\{x \in A \mid \phi(x)\} \in \mathcal{P}(A)
$$

Moreover, two equiprovable small propositional functions give rise to the same subset, that is in emTT we can derive

$$
\frac{\phi_{1}(x) \leftrightarrow \phi_{2}(x) \text { true }[x \in A]}{\left\{x \in A \mid \phi_{1}(x)\right\}=_{\mathcal{P}(A)}\left\{x \in A \mid \phi_{2}(x)\right\} \text { true }}
$$

In the following we indicate subsets of a set $A$ with capital letters $U, V, W \ldots$

Associated with the notion of subset we have also a subset membership indicated with the symbol $\epsilon$, which we distinguish from the primitive set membership $\in$ used to say that an element belongs to a

\footnotetext{
${ }^{2}$ We formally restrict collection constructors to those present in the fragment emTT 0 in [Mai12].

${ }^{3}$ The notation $A \rightarrow \mathcal{P}(1)$ for the power-collection $\mathcal{P}(A)$ is used to remember that its elements are operations from a set $A$ to the power-collection on the singleton.
} 
certain set. Given a subset $U \subseteq A$ of a set $A$, i.e. $U \in \mathcal{P}(A)$, for any $a \in A$ we define a new small proposition

$$
a \epsilon U \operatorname{prop}_{s} \text {. }
$$

We can prove in emTT that

$$
U=\{x \in A \mid x \in U\} \in \mathcal{P}(A)
$$

and also that, for any small propositional function $\phi(x) \in \operatorname{prop}_{\mathrm{s}}[x \in A]$ on the set $A$ and for any element $a \in A$,

$$
a \epsilon\{x \in A \mid \phi(x)\} \leftrightarrow \phi(a) \text { true } .
$$

The subset equality is equivalent to usual extensional equality with respect to membership $\epsilon$, namely we can derive in emTT that

$$
\forall x \in A(x \in U \leftrightarrow x \in W) \leftrightarrow U=_{\mathcal{P}(A)} W \text { true }
$$

and, of course, that

$$
\{x \in A \mid \phi(x)\}=_{\mathcal{P}(A)}\{x \in A \mid \psi(x)\} \quad \leftrightarrow \quad \forall_{x \in A}(\phi(x) \leftrightarrow \psi(x)) \text { true. }
$$

In particular, $\mathcal{P}(1)$ denotes the power-collection of the singleton $N_{1}$, and its elements are equivalence classes of small propositions closed under the equivalence relation of equiprovability.

The fact that subset equality corresponds to usual extensional equality of sets suggests that we can view the subset theory in emTT as a local set theory where subsets of a set $A$ can be considered local sets [Bel88] in the style of Zermelo-Fraenkel set theory. Then, membership and extensional equality via elements becomes a local property restricted to a given set $A$. To this purpose, observe that among subsets of $A$, there is $A$ itself thought of as the subset

$$
\{x \in A \mid \text { tt }\}
$$

where tt is any tautology. Moreover, we can define quantifiers relativized to a subset; this means that, if $U \subseteq A$ and $\varphi$ is a propositional function with an argument in $A$, we write $\exists x \epsilon U \varphi$ as an abbreviation for $\exists x \in A(x \in U \& \varphi)$, and $\forall x \in U \varphi$ as an abbreviation for $\forall x \in A(x \in U \rightarrow \varphi)$. A consequence of these definitions is that all laws of many-sorted intuitionistic logic regarding quantifiers extend to quantifiers relativized to a subset.

Note that the membership relation $\epsilon$ between subsets is crucial in emTT to obtain an embedding of subsets into sets, which associates the set

$$
\Sigma_{x \in A} x \in U \text { set }
$$

with a subset $U \subseteq A$. In this way an operation from $U \subseteq A$ to a set $B$ can be represented as an operation in $\Sigma_{x \in A} x \in U \rightarrow B$.

The emTT-distinction between set and collection is analogous to the distinction between set and class in axiomatic set theory. But while in axiomatic set theory the distinction is mainly due to problems with consistency (or size), here it is motivated by quality of information and preservation of predicativity. Indeed, sets are kept distinct from collections to be able to keep a distinction between computable, effective domains (represented by sets) and non computable ones (represented by collections). According to this, only proofs of small propositions can be inductively generated to form a set. Instead proofs of arbitrary propositions just form a collection because they may include quantifications over proper collections and hence they cannot be inductively generated in general. Then, to avoid an impredicative power-collection of a set, a subset must be defined as an equivalence class of small propositional functions and not of generic ones.

An important conceptual reason why even the power-collection $\mathcal{P}(1)$ of the singleton is only a collection and not a set is that in emTT we intend the notion of small proposition as open. The same we do for that of proposition, of set and of collection. Indeed, whilst we have fixed the system emTT, new sets or collections can be introduced at any time. This implies in particular that the collection of small propositions (quotiented under equiprovability), or that of sets, is not a set. Indeed, each time we fix 
our propositions or sets by fixing a formal system, both notions become inductively generated. However, we cannot support an induction principle inside the formal system, given that the number of inductive hypotheses should change any time we introduce a new set or proposition. This is different from the induction principle on the set of natural numbers, which has only two hypothesis: what we do on the number zero, and with any successor number.

\subsection{Benefits of distinguishing operations from functions}

Inspired by Brouwer's difference between lawlike and choice sequences [Tv88], we can define choice sequences from the set of natural numbers $\mathrm{N}$ to a set $B$ as functions (in the sense of functional relations, that is, total and single-valued relations), and lawlike sequences as operations:

Def. 3.1 (choice and lawlike sequences) Given a set $A$, a choice sequence from the set $N$ of natural numbers to $A$ is a function defined by a small functional relation $\alpha(x, y) \operatorname{prop}_{s}[x \in N, y \in A]$ in emTT.

$A$ lawlike sequence from the set $N$ of natural numbers to $A$ is an operation

$$
f \in N \rightarrow A
$$

in emTT, or equivalently, thanks to the rules in [Mai09] defining elements in $N \rightarrow A$, an emTT-term $f(x) \in A[x \in N]$.

It is possible to keep a distinction between choice sequences and lawlike sequences because in emTT the axiom of unique choice

$$
\left(\mathrm{AC} !_{N, N}\right) \quad \forall x \in \mathrm{N} \exists ! y \in \mathrm{N} R(x, y) \quad \longrightarrow \quad \exists f \in \mathrm{N} \rightarrow \mathrm{N} \forall x \in \mathrm{N} R(x, f(x)),
$$

which turns a function between natural numbers into an operation, is not valid, as shown in [Mai12 $]^{4}$. Our distinction allows us to clarify and compare results about choice sequences in the literature, since choice sequences are sometimes identified with our functions [Rat05], sometimes with our operations [Tv88].

Another consequence of the distinction between operations and functions is that we can refine the notion of decidable subset of the set of natural numbers N. In constructive mathematics it is common to say that a subset $U \subseteq \mathrm{N}$ is decidable if $\forall x(x \epsilon U \vee x \epsilon \neg U)$ holds. In our theory we can distinguish three notions:

Def. 3.2 A subset $U$ of the set $N$ is said to be:

- complemented, if $\forall x(x \in U \vee \neg(x \in U))$ holds. In this case $U$ is classified by a function from $N$ to the boolean set Bool

$$
\chi_{U}(x, y) \equiv\left(x \in U \& y={ }_{\text {Bool }} 1\right) \vee\left(x \in U \& y={ }_{\text {Bool }} 0\right)
$$

- detachable, if the subset $U$ is classified by an operation, namely we can derive

$$
\exists_{f \in N \rightarrow \text { Bool }} \forall x \in N\left(\left(x \in U \& f(x)={ }_{\text {Bool }} 1\right) \vee\left(x \in U \& f(x)={ }_{\text {Bool }} 0\right)\right)
$$

- decidable, if $U$ is classified by a computable operation, namely we can derive

$$
\begin{array}{r}
\exists_{f \in N \rightarrow \text { Bool }}\left(\forall x \in N \left(\left(x \in U \& f(x)={ }_{\text {Bool } 1)} \vee\left(x \notin U \& f(x)=\text { Bool }_{0}\right)\right)\right.\right. \\
\left.\& \exists e \in N \quad \forall x \in N \exists y \in N\left(T(e, x, y) \wedge U(y)={ }_{N} f(x)\right)\right)
\end{array}
$$

where $T(e, x, y)$ is the Kleene predicate expressing that $y$ is the computation executed by the program numbered $e$ on the input $x$ and $U(y)$ is output of the computation $y$.

\footnotetext{
${ }^{4}$ The version of emTT presented here corresponds to the version of $\mathrm{emTT}_{0}$ in [Mai12].
} 
Observe that, classically, all subsets are complemented. Of course, in the presence of the axiom of unique choice, functions and operations coincide and hence complemented and detachable subsets coincide, too, as for example in Martin-Löf's type theory.

All the three kinds of subsets coincide in the Kleene realizability interpretation of Heyting arithmetic. This interpretation is in some sense the intended interpretation of the arithmetic fragment of a constructive foundation. Hence, the identification of the name "decidable" with our notion of complemented subsets, (that we do not follow here, though) has its own (plausible) justification.

In [Mai12], we observe that if we extend emTT with the principle of excluded middle then we can prove the existence of a power-set of detachable subsets, which do not necessarily coincide with all subsets, i.e. with complemented ones. This option of restricting exponentiation as a set to lawlike sequences opens the way to build a theory compatible with classical predicativity as in Feferman's theories [Fef79].

\section{The need of pointfree topology}

We now explain why in order to develop topology in the minimalist foundation, the pointfree approach is a necessity and not an option. The most important reason is that, when working in the minimalist foundation, the point-wise approach is not suitable because relevant examples of classical topologies (real numbers both as Dedekind cuts or Cauchy sequences, Baire space, Cantor space,...) do not give rise to a point-wise topology since their points do not form a set.

A solution is to work with the pointfree topology associated to each of these spaces. The constructive approach to pointfree topology given by formal topology has provided evidence that most important results of constructive analysis (see for example [Mar70, Pal05b]) can be reached without assuming further principles, such as the Fan Theorem adopted by Brouwer in his point-wise approach [BR87, Tv88, Ish05, Bri08].

Before entering into details, we briefly review a constructive notion of topological space and then the main concepts of formal topology.

\subsection{A predicative and intuitionistic notion of topological space}

Considering that in a predicative foundation the discrete topology on a given non-empty set is not a set but a collection, we need to review the concept of topological space by distinguishing what belongs to the realm of sets from what belongs to the realm of collections.

At first, one could think of simply keeping the traditional definition of topological space $(X, \mathcal{O} X)$ by just declaring the topology $\mathcal{O} X$ to be only a subcollection of the power of $X$ which is a suplattice, i.e. a complete join-semilattice, with finite distributive meets. This approach is compulsory in order to include the discrete topology among topologies. Even more, as shown in [Cur10], there is no non-trivial suplattice, and hence no non-trivial topology, which is a set.

One should then define suplattices as collections closed under sups of set-indexed families. However, as in [Sam87] and [BS06], suplattices are easier to handle by restricting to the notion of set-based suplattice, namely a semilattice that is generated by taking sups from a set(-indexed family) of elements, called generators.

Topologically this means that we need to assume that the collection of opens of a space has a base that is a set. To make this assumption rigorous, we require that for a given set of points $X$ we have a set $S$ together with a family of subsets ext $(a) \subseteq X[a \in S]$ acting as a base for the topology on $X$. Elements $a$ of $S$ act as names of basic opens of $X$; they are called formal basic neighbourhoods or simply observables.

Then, following [Sam03], we define a subset of $X$ to be open if it is equal to ext $U \equiv \cup_{a \epsilon U}$ ext $a$ for some subset $U \subseteq S$. It is immediate to see that open subsets are closed under unions of set-indexed families.

Then we need to require closure of open subsets under intersection. To this purpose, it is convenient to start from basic neighbourhoods, that is subsets of $X$ of the form ext $a$ for some $a \in S$. For all $a, b \in S$, the intersection ext $a \cap \operatorname{ext} b$ is open, that is, it is equal to ext $W$ for some $W \subseteq S$, if and only if 
where

$$
a \downarrow b \equiv\{c \in S: \operatorname{ext} c \subseteq \operatorname{ext} a \cap \operatorname{ext} b\}
$$

In fact, ext $(a \downarrow b)$ is by its definition the greatest open subset contained in ext $a \cap$ ext $b$. Then, from B0, by two applications of distributivity in $\mathcal{P} X$, we can easily obtain

B1 ext $U \cap \operatorname{ext} V=\operatorname{ext}(U \downarrow V)$ for all $U, V \subseteq S$,

where

$$
U \downarrow V \equiv \cup_{a \epsilon U} \cup_{b \epsilon V} a \downarrow b .
$$

Finally, to obtain that the whole space is open we need to add the requirement

$$
\text { B2 } X=\operatorname{ext} S \text {. }
$$

It is clear that for any family of subsets of $X$ indexed by the set $S$, i.e. for any ext $a \subseteq X$ for $a \in S$, satisfying B1 and B2 the collection of subsets ext $U \subseteq X$ for $U \in \mathcal{P} S$ is closed under set-indexed unions and finite intersections.

Therefore we can give the following constructive version of topological spaces (see [Sam03]):

Def. 4.1 $A$ concrete space is a structure $\mathcal{X}=(X$, ext, $S)$ where $X, S$ are sets and ext $(a) \subseteq X[a \in S]$ is a set-indexed family of subsets satisfying:

$$
\begin{aligned}
& \text { B1 } \operatorname{ext} U \cap \operatorname{ext} V=\operatorname{ext}(U \downarrow V) \text { for all } U, V \subseteq S, \\
& \text { B2 } X=\operatorname{ext} S .
\end{aligned}
$$

In an impredicative foundation with powersets, this is just a reformulation of the common notion of topological space.

The notion of concrete space is present in Bishop [Bis67], under the name of neighbourhood space. The discrete topology on a set $X$ is obviously an example of concrete space with $X$ itself as base and $\operatorname{ext}(x) \equiv\{x\}$ for $x \in X$.

A useful example of concrete space is given by the set $\mathbb{Q}$ of rational numbers with the topology produced by the base of open intervals. In more detail, the base is the set $\mathbb{Q} \times \mathbb{Q}$ of pairs $\langle p, q\rangle$ of rational numbers, and the basic neighbourhood with index $\langle p, q\rangle$ is the subset

$$
\operatorname{ext}(\{(p, q)\}) \equiv\{r \in \mathbb{Q} \mid p<r<q\}
$$

for all $p, q \in \mathbb{Q}$.

In other intuitionistic and predicative foundations, as Aczel's CZF and Martin-Löf's type theory in [NPS90], another example of concrete space is that of real numbers. It is not so in our minimalist foundation, as we shall see later. Even when the topology of real numbers provides an example of concrete space, it is well known from Brouwer that a constructive pointwise development of analysis fails to get important properties [Tv88], as compactness of the closed interval [0,1], unless further principles, as the Fan Theorem, are assumed or some basic topological notions are changed as in Bishop's approach (see [Bis67, BB85, BR87]). An alternative approach to constructive topology, and analysis, is offered by formal topology.

\subsection{The pointfree approach of formal topology}

The approach of formal topology to pointfree topology was introduced by Per Martin-Löf and the second author in the 80s; the first published account is [Sam87]. The intended foundation was then Martin-Löf's type theory MLTT in [NPS90]. However, as underlined in the introduction of [Sam87], to the second author it was already clear that it was necessary to work with an explicit notion of subset, and with a primitive notion of proposition using the judgement that a proposition is true without any reference to proof-terms in [Mar85]. Such a conception of subsets and propositions was later specified in [SV98] as a tool to be added on top of type theory. As noticed in [Mai98, Mai05b], working with existential 
quantifiers with no proof terms means that the axiom of choice no longer holds. This is different from MLTT where existential quantifications are identified with indexed sums, according to the propositionas-set isomorphism, thus making the axiom of choice derivable (see [Mar84]). Moreover, this explains why in formal topology, as developed by the second author, every use of the axiom of choice was explicit. Given that the notion of subset in [SV98] and a primitive notion of proof-irrelevant propositions have been incorporated in our minimalist foundation, all the main definitions and results on formal topology (by the second author) can be carried in it. Actually the combination of the tool of extensional subsets with the intensional MLTT partly anticipated the notion of two-level theory in [MS05], because subsets are not formally included in MLTT.

The main idea of formal topology is to replace the notion of concrete space with an abstract axiomatization of the structure of open subsets, and then to recover its points in a formal way as suitable subsets of opens. The precise definition is reached by describing the structure of the set $S$ of basic neighbourhoods in a concrete space $(X$, ext,$S)$ with no mention of the set $X$.

While in the concept of concrete space $(X$, ext,$S)$ points in $X$ are given in the same time with the formal basic neighbourhoods in $S$ and both form a set, in formal topology only the structure of opens is described starting from the set $S$ of formal basic neighbourhoods and from a new primitive relation $a \triangleleft U$, called formal cover, between formal basic neighbourhoods $a \in S$ and subsets $U \subseteq S$. A formal cover relation is the abstract counterpart of

$$
\operatorname{ext} a \subseteq \operatorname{ext} U
$$

which expresses in a concrete space that the open ext $U$ is a covering of the basic neighbourhood ext $a$. Then, the notion of formal topology extends that of formal cover with the addition of a primitive predicate $\operatorname{Pos}(a)$ for $a \in S$, which is the abstract counterpart of the assertion that the basic neighbourhood ext $a$ is inhabited. Details of the definitions are now presented.

Def. 4.2 (formal cover) $A$ formal cover $\mathcal{A}=(S, \triangleleft)$ is given by a set $S$ and a relation $\triangleleft \subseteq S \times \mathcal{P}(S)$ between elements and subsets of $S$ that satisfies the following rules for every $a \in S$ and $U, V \subseteq S$ :

$$
\frac{a \epsilon U}{a \triangleleft U} \text { reflexivity } \quad \frac{a \triangleleft U \quad U \triangleleft V}{a \triangleleft V} \text { transitivity } \quad \frac{a \triangleleft U \quad a \triangleleft V}{a \triangleleft U \downarrow \downarrow_{\mathcal{A}} V} \text { convergence }
$$

where $U \triangleleft V \stackrel{\text { def }}{\Longleftrightarrow}(\forall b \epsilon U)(b \triangleleft V)$ and $U \downarrow_{\mathcal{A}} V=\{a \in S:(\exists u \epsilon U)(a \triangleleft u) \&(\exists v \epsilon V)(a \triangleleft v)\}$.

This definition provides a predicative counterpart of the impredicate notion of point-free topology called locale [MM92, Joh82]. In fact, to any formal cover $\mathcal{A}=(S, \triangleleft)$ we can associate an operator $\mathcal{A}$ on $\mathcal{P}(S)$, i.e. a map $\mathcal{A}: \mathcal{P}(S) \rightarrow \mathcal{P}(S)$ (that by abuse of notation we call as the formal cover itself!), by putting

$$
\mathcal{A} U \stackrel{\text { def }}{=}\{a \in S \mid a \triangleleft U\} .
$$

for any $U \subseteq S$. Then, reflexivity and transitivity of the cover means that the operator $\mathcal{A}$ is a saturation (or closure operator) and convergence means that $\mathcal{A}$ satisfies

$$
\mathcal{A}\left(U \downarrow_{\mathcal{A}} V\right)=\mathcal{A} U \cap \mathcal{A} V
$$

The collection $\operatorname{Sat}(\mathcal{A})$ of all fixed points of the operator $\mathcal{A}$ (i.e. all subsets $U$ of $S$ satisfying $\mathcal{A}(U)=U)$ with the order given by inclusion forms a locale. See [CMS13] for an account and discussion on the several variants of the definitions of formal cover.

Then a formal topology is defined as follows:

Def. 4.3 A formal topology $\mathcal{S}=(S, \triangleleft$, Pos $)$ is a formal cover $(S, \triangleleft)$ equipped with a positivity predicate, that is a predicate $\operatorname{Pos}(a)$ for $a \in S$ which satisfies the conditions

$$
\text { (monotonicity) } \frac{\operatorname{Pos}(a) \quad a \triangleleft U}{(\exists u \epsilon U) \operatorname{Pos}(u)} \quad \text { (positivity) } \frac{\operatorname{Pos}(a) \rightarrow a \triangleleft U}{a \triangleleft U}
$$


Formal topologies provides a predicative counterpart of the impredicative notion of open locale [JT84].

Formal covers, as well as formal topologies, can be inductively generated from a set-indexed family of axioms of the form $a \triangleleft U$ :

Def. 4.4 Given a set $S$, an axiom-set is a pair $I, C$, given by a family of sets $I(a)$ for each a $\in S$ and a family of subsets $C(a, i) \subseteq S$ for $a \in S$ and $i \in I(a)$ with the intended meaning that $a \triangleleft C(a, i)$ holds.

The definition of inductively generated formal cover was introduced in [CSSV03] and for our purposes we just recall that:

Def. 4.5 Given a pre-ordered set $(S, \leq)$ and an axiom-set $I, C$, the inductively generated formal cover (formal topology) $\left(S, \triangleleft_{I, C}\right)$ is a formal cover (formal topology) satisfying:

(i) $a \triangleleft_{I, C} C(a, i)$ for every $a \in S$ and $i \in I(a)$;

(ii) if $\triangleleft^{\prime}$ is another formal cover (formal topology) such that $a \triangleleft^{\prime} C(a, i)$ for all $a \in S$ and $i \in I(A)$, then $a \triangleleft_{I, C} U \rightarrow a \triangleleft^{\prime} U$ holds for all $a \in S$ and $U \subseteq S$.

In the minimalist foundation we assume the existence of an inductively generated cover when needed. See [Val07] for a proof on how to build inductively generated formal covers, and formal topologies, in an extension of Martin-Löf's type theory with ordinals.

We now recall the notion of formal point. Given any formal topology $\mathcal{S}$, a formal point over $\mathcal{S}$ is a subset $\alpha$ of the set $S$ such that it makes sense to think of $a \epsilon \alpha$ as meaning that the observable $a$ is an approximation of $\alpha$. To obtain a precise definition, one considers the case in which $\mathcal{S}$ is the topology of a concrete space $X$ and takes the pointfree properties of the subset $\{a \in A \mid x \epsilon$ ext $(a)\}$, which is the trace on $S$ of a concrete point $x \in X$, as the conditions to define a subset $\alpha \subseteq S$ to be a formal point.

Def. 4.6 (Formal point) Let $\mathcal{A} \equiv(A, \triangleleft)$ be a formal cover. Then an inhabited subset $\alpha$ of $A$ is a formal point if, for any $a, b \in A$ and any $U \subseteq A$, it satisfies the following conditions:

$$
\text { ( } \alpha \text { is filtering) } \frac{a \epsilon \alpha \quad b \epsilon \alpha}{\left(\exists c \epsilon\{a\} \downarrow_{\mathcal{A}}\{b\}\right) c \epsilon \alpha} \quad\left(\alpha \text { splits the cover) } \frac{a \epsilon \alpha \quad a \triangleleft U}{(\exists u \epsilon U) u \epsilon \alpha}\right.
$$

Then, one can take the collection of $\operatorname{Pt}(\mathcal{A}) \equiv\{\alpha \in \mathcal{P}(S) \mid \alpha$ formal point $\}$ and make it a formal space as follows:

Def. 4.7 (formal space) For any formal cover $\mathcal{A} \equiv(S, \triangleleft)$, the collection $\operatorname{Pt}(\mathcal{A})$ of formal points of $\mathcal{A}$ with the topology generated by the basic neighbourhoods of the form $\operatorname{Ext}(a) \equiv\{\alpha \in \operatorname{Pt}(\mathcal{A}) \mid a \epsilon \alpha\}$ for $a \in S$ defines the formal space of points of $\mathcal{A}$ (that by abuse of notation we still call $\operatorname{Pt}(\mathcal{A})$ ).

In an impredicative foundation, where power-collections are sets, it is clear that $\operatorname{Pt}(\mathcal{A})$ defines a concrete space for any formal cover $\mathcal{A}$. Hence, as it is well known, impredicatively one can prove the existence of an adjunction between formal covers and concrete spaces [Joh82, MM92]. This impredicative adjunction associates to a formal cover its formal space, and conversely to a concrete space $(X$, ext, $S)$ the formal cover $\left(S, \triangleleft_{X}\right)$ defined by

$$
a \triangleleft_{X} U \equiv \operatorname{ext} a \subseteq \operatorname{ext} U
$$

But, not all formal covers arise from concrete spaces in this way. Moreover, the formal cover induced by a formal space is not necessarily equal to the starting formal cover $\mathcal{A}$, i.e. not all formal covers are spatial. And even more the formal space of a formal cover arising from a concrete space is not necessarily equivalent to the starting concrete space, i.e. not all concrete spaces are sober.

In a constructive and predicative foundation as our minimalist one, such an adjunction is no longer available, because the collection $\operatorname{Pt}(\mathcal{A})$ is not necessarily a set.

Here we will see at least three relevant examples of proper formal spaces, i.e. formal spaces whose formal points cannot form a set in our minimalist foundation: the formal space of real numbers, Cantor and Baire spaces. In all these examples, we will see how our foundation allows to distinguish points which are given effectively, i.e. concrete points identified with lawlike sequences, from points which are only ideally so, i.e. formal points, which are identified with choice sequences. It is a predicative 
foundation which allows one, and, in the same time compels one, to take care of this distinction between an effective or real structure, as that of opens, from an ideal or non effective structure as that of formal points.

So in a constructive approach to topology as our minimalist one, formal topologies and formal points are not just an option to describe something which is there in any case. They are introduced as the only way to treat also those spaces which otherwise would be constructively unreachable.

\subsection{Examples of point-free topologies whose formal points do not form a set}

The first example of topology whose formal points do not form a set in the minimalist foundation is the formal topology of real numbers as Dedekind cuts:

Def. 4.8 (Formal topology of real numbers) The formal topology of real numbers $\mathcal{R} \equiv(\mathbb{Q} \times$ $\left.\mathbb{Q}, \triangleleft_{\mathcal{R}}, \operatorname{Pos}_{\mathcal{R}}\right)$ is an inductively generated formal topology defined as follows. The base is $\mathbb{Q} \times \mathbb{Q}$ and the basic neighbourhoods are pairs of rational numbers, $\langle p, q\rangle$ with $p, q \in \mathbb{Q}$. A preorder on $\mathbb{Q} \times \mathbb{Q}$ is defined as follows

$$
\langle p, q\rangle \leq\left\langle p^{\prime}, q^{\prime}\right\rangle \equiv p^{\prime} \leq p \leq q \leq q^{\prime}
$$

for $p, q, p^{\prime}, q^{\prime}$ in $\mathbb{Q}$. The cover is defined inductively by the following rules (which are a formulation in our context of Joyal axioms, cf. [Joh82], pp. 123-124):

$$
\begin{aligned}
& \frac{q \leq p}{\langle p, q\rangle \triangleleft_{\mathcal{R}} U} \quad \frac{\langle p, q\rangle \in U}{\langle p, q\rangle \triangleleft_{\mathcal{R}} U} \quad \frac{p^{\prime} \leq p<q \leq q^{\prime} \quad\left\langle p^{\prime}, q^{\prime}\right\rangle \triangleleft_{\mathcal{R}} U}{\langle p, q\rangle \triangleleft_{\mathcal{R}} U} \\
& \frac{p \leq r<s \leq q \quad\langle p, s\rangle \triangleleft_{\mathcal{R}} U \quad\langle r, q\rangle \triangleleft_{\mathcal{R}} U}{\langle p, q\rangle \triangleleft_{\mathcal{R}} U} \quad \text { wc } \frac{w c(\langle p, q\rangle) \triangleleft_{\mathcal{R}} U}{\langle p, q\rangle \triangleleft_{\mathcal{R}} U}
\end{aligned}
$$

where in the last axiom we have used the abbreviation

$$
w c(\langle p, q\rangle) \equiv\left\{\left\langle p^{\prime}, q^{\prime}\right\rangle \in \mathbb{Q} \times \mathbb{Q} \mid p<p^{\prime}<q^{\prime}<q\right\}
$$

(wc stands for 'well-covered').

The positivity predicate is $\operatorname{Pos}_{\mathcal{R}}(\langle p, q\rangle) \equiv p<q$, expressing that the pair of rationals represents a non-empty interval.

As shown in [NS99], formal points of the formal topology $\mathcal{R}$ are in bijection with the collection of Dedekind cuts on the rationals. The proof carries over to our foundation.

Def. 4.9 $A$ Dedekind cut on the rationals is a pair $(L, U)$ with inhabited $L, U \subseteq \mathbb{Q}$ satisfying the following properties:

$$
\begin{array}{ll}
\text { (disjointness) } & \forall q \in \mathbb{Q} \neg(q \in U \& q \in L) \\
\text { (L-openess) } & \forall p \in L \quad \exists q \in L p<q \\
\text { (U-openess) } & \forall q \in U \quad \exists p \in U p<q \\
\text { (L-monotonicity) } & \forall q \in L \forall p \in \mathbb{Q}(p<q \rightarrow p \epsilon L) \\
\text { (U-monotonicity) } & \forall p \in U \forall q \in \mathbb{Q}(p<q \rightarrow q \epsilon U) \\
\text { (locatedness) } & \forall q \in \mathbb{Q} \forall p \in \mathbb{Q}(p<q \rightarrow p \in L \vee q \in U)
\end{array}
$$

Proposition 4.10 In emTT $+\triangleleft_{\mathcal{R}}$, formal points of the inductively generated formal topology $\mathcal{R}$ are in bijection with the collection of Dedekind cuts on the rationals.

Proof. Given a formal point $\alpha \in \operatorname{Pt}(\mathcal{R})$ we can build the following Dedekind cut:

$$
L_{\alpha} \equiv\{p \in \mathbb{Q} \mid\langle p, q\rangle \epsilon \alpha\} \quad U_{\alpha} \equiv\{q \in \mathbb{Q} \mid\langle p, q\rangle \epsilon \alpha\}^{5}
$$

Conversely, given a Dedekind cut $(L, U)$ we can define the following formal point

$$
\alpha_{(L, U)} \equiv\{\langle p, q\rangle \in \mathbb{Q} \times \mathbb{Q} \mid p \in L \& q \in U\}
$$

\footnotetext{
${ }^{5}$ Note that the base of our topology $\mathcal{R}$ does not contain $+\infty,-\infty$ as that in [NS99].
} 
In [NS99] it is proved that formal points of $\mathcal{R}$, or Dedekind cuts, are also in bijective correspondence with Cauchy sequences à la Bishop [Bis67]. This correspondence does not work in emTT: only Cauchy sequences à la Bishop can be shown to be formal points of $\mathcal{R}$. To make this point clear, we recall the notion of Cauchy sequence à la Bishop. In the following with $\mathrm{N}^{+}$we mean the set of positive natural numbers.

Def. 4.11 (Cauchy sequence à la Bishop) A function $R(x, y) \operatorname{prop}_{s}\left[x \in N^{+}, y \in \mathbb{Q}\right]$, indicated with the usual notation $\left(x_{n}\right)_{x \in N^{+}}$, is a Cauchy sequence in emTT if we can prove for any $n, m \in N^{+}$

$$
\left|x_{n}-x_{m}\right| \leq 1 / n+1 / m^{6}
$$

As in [NS99], also in emTT we can prove that any Cauchy sequence $\left(x_{n}\right)_{x \in \mathrm{N}}$ determines a formal point $\alpha$ of the formal topology $\mathcal{R}$ if we define it by:

$$
\alpha \equiv\left\{\langle p, q\rangle \in \mathbb{Q} \times \mathbb{Q} \mid \exists n \in \mathrm{N}^{+} p<x_{n}-2 / n<x_{n}+2 / n<q\right\}
$$

Conversely, given a formal point $\alpha$, we can prove in emTT that within $\alpha$ there exists a countable number of strictly decreasing intervals as follows

$$
\begin{array}{r}
\forall n \in \mathrm{N}^{+} \exists\left\langle x_{n}, y_{n}\right\rangle \in \mathbb{Q} \times \mathbb{Q}\left(\left(\left\langle x_{n}, y_{n}\right\rangle \in \alpha \quad \& \quad\left|x_{n}-y_{n}\right|<(2 / 3)^{n}\right)\right. \\
\& \exists\left\langle x_{n+1}, y_{n+1}\right\rangle \in \mathbb{Q} \times \mathbb{Q} \quad\left(x_{n} \leq x_{n+1}<y_{n+1} \leq y_{n} \quad \&\right. \\
\left.\left.\left(\left\langle x_{n+1}, y_{n+1}\right\rangle \in \alpha \quad \& \quad\left|x_{n+1}-y_{n+1}\right|<(2 / 3)^{n+1}\right)\right)\right)
\end{array}
$$

This is proved by induction; in fact, for $n \in \mathrm{N}$ and $\left\langle x_{n}, y_{n}\right\rangle \epsilon \alpha$ we can find a covering

$$
\left\langle x_{n}, y_{n}\right\rangle \triangleleft_{\mathcal{R}}\left\{l_{n_{1}}, l_{n_{2}}\right\}
$$

such that $l_{n_{1}} \equiv\left\langle x_{n}, z_{n_{1}}\right\rangle$ and $l_{n_{2}} \equiv\left\langle z_{n_{2}}, y_{n}\right\rangle \quad$ with $\quad z_{n_{1}} \equiv x_{n}+\left(2 \cdot\left(y_{n}-x_{n}\right)\right) / 3$ and $z_{n_{2}} \equiv$ $x_{n}+\left(y_{n}-x_{n}\right) / 3$. Since the formal point $\alpha$ splits the cover, we can prove

$$
\forall n \in N \quad \exists i=1,2 \quad l_{n i} \epsilon \alpha .
$$

However, such $l_{n i}$ is not necessarily unique, because the formal point can be cointained in both!

Classically, one can define a function $\mathcal{L}(n)$ for $n \in N$ by cases by putting

$$
\mathcal{L}(n) \equiv \begin{cases}l_{n 1} & \text { if } l_{n_{1}} \in \alpha \\ l_{n 2} & \text { if } l_{n_{2}} \in \alpha \& \neg l_{n_{1}} \in \alpha\end{cases}
$$

Constructively this does not work because $\alpha$ is not complemented. But, if we work in a foundation as Martin-Löf's type theory MLTT, actually in the setoid model over it, by using the axiom of dependent choice on $\mathbb{Q} \times \mathbb{Q}$ we can even extract an operation $l(n) \in \mathbb{Q} \times \mathbb{Q}\left[n \in \mathrm{N}^{+}\right]$such that for any $n \in \mathrm{N}^{+}$

$$
l(n) \epsilon \alpha
$$

and, after naming $l(n)={ }_{Q \times Q}\left\langle x_{n}, y_{n}\right\rangle$ the values of the operation on each natural number, the conditions $x_{n}-y_{n} \leq(2 / 3)^{n}$ and $x_{n} \leq x_{n+1}<y_{n+1} \leq y_{n}$ hold for each natural number $n$. Then, a Cauchy sequence can be defined by taking the first components $\left(x_{n}\right)_{n \in N}$ or the second components $\left(y_{n}\right)_{n \in N}$. Hence, any Dedekind cut or formal point of $\mathcal{R}$ corresponds to a lawlike Cauchy sequence à la Bishop in MLTT.

Since in our foundation no axiom of choice is available, this proof cannot be carried out. At a closer look, it does not appear constructively justified to be able to extract a choice of the interval where the formal point is, with no extra information.

What actually happens in MLTT is that the splitting of points is already given with an operation choosing an interval where the point is, and hence from this choice a definition by cases can be given similarly to that done classically. This example explains why in Martin-Löf's type theory real numbers

\footnotetext{
${ }^{6}$ This is formally written as $\forall p \in \mathbb{Q} \forall q \in \mathbb{Q}(R(n, p) \& R(m, q) \rightarrow|q-p| \leq 1 / n+1 / m)$ where the definition of module is the usual one.
} 
as formal points are only the lawlike ones, namely those for which we can extract a lawlike Cauchy sequence.

This is not true in our foundation. In fact, the property that a formal point splits the cover is expressed through an existential quantifier $\exists_{x \in A} \phi(x)[w \in \Gamma]$ under a context $\Gamma$, which does not necessarily provide an operation $\operatorname{wit}(d) \in A[w \in \Gamma]$ depending on the context. Such an operation is available only in a Kleene realizability interpretation of our foundation. As expected, in emTT real numbers as formal points of the formal topology $\mathcal{R}$ cannot coincide with lawlike Cauchy sequences, as shown in [Mai12]. Even more, as proved in [Mai12], real numbers as formal points of $\mathcal{R}$, and hence as Dedekind cuts, do not form a set. Analogously, also real numbers as Cauchy sequences à la Bishop do not form a set. These results are obtained through a realizability interpretation of emTT which interprets emTT-sets as countable subsets of natural numbers and emTT-collections as entities which are not necessarily countable.

Now we describe two other examples of formal topologies whose formal points do not form a set in emTT. These are Cantor and Baire formal topologies, which are defined as instances of the more general notion of formal topology on the tree over a set. In order to define such formal topologies, we need to represent the tree over a set $A$, which we identify with the nodes labelled by lists of elements in a set $A$, using the abbreviation $A^{*} \equiv \operatorname{List}(A)$. We write $[l, x]$ for the list obtained by appending $x \in A$ to the list $l \in A^{*}$ and $[l, t]$ for the list obtained by appending the list $t \in A^{*}$ to the list $l \in A^{*}$.

Def. 4.12 The tree formal topology over a set $A$ is the formal topology $A^{N} \equiv\left(A^{*}, \triangleleft_{A^{N}}\right.$, Pos $\left._{A^{N}}\right)$ where $\triangleleft_{A^{N}}$ is inductively generated by the following rules

$$
r f l \frac{l \epsilon V}{l \triangleleft_{A^{N}} V} \quad \leq \frac{s \sqsubseteq l \quad l \triangleleft_{A^{N}} V}{s \triangleleft_{A^{N}} V} \quad \operatorname{tr} \frac{\forall x \in A[l, x] \triangleleft_{A^{N}} V}{l \triangleleft_{A^{N}} V}
$$

where $s \sqsubseteq l \equiv \exists_{t \in A^{*}} s=_{A^{*}}[l, t]$, i.e. $l$ is an initial segment of $s$.

The positivity predicate is true on any element, i.e. $\operatorname{Pos}_{A^{N}}(l) \equiv \mathrm{tt}$ for any $l \in A^{*}$.

Among tree formal topologies, we distinguish Cantor and Baire formal topologies as follows:

Def. 4.13 (Cantor and Baire formal topologies) The tree formal topology when $A \equiv\{0,1\}$, namely

$$
\{0,1\}^{N} \equiv\left(\{0,1\}^{*}, \triangleleft_{\{0,1\}^{N}}, \operatorname{Pos}_{\{0,1\}^{N}}\right)
$$

is called Cantor formal topology.

The tree formal topology when $A \equiv N$, namely

$$
N^{N} \equiv\left(N^{*}, \triangleleft_{N^{N}}, \operatorname{Pos}_{N^{N}}\right)
$$

is called Baire formal topology.

Formal points of such topologies coincide with choice sequences of definition 3.1:

Proposition 4.14 Formal points $\operatorname{Pt}\left(A^{N}\right)$ of the tree formal topology over a set $A$ are in bijective correspondence with choice sequences on the tree $A^{*}$.

Proof. Given a formal point $\alpha$, we define a function $R_{\alpha}(x, y) \operatorname{prop}_{s}[x \in \mathrm{N}, y \in A]$ as follows:

$$
R_{\alpha}(n, a) \equiv \exists l \in \alpha \quad l_{n+1}={ }_{A} a
$$

where $l_{n}$ is the n-th component of $l$.

Conversely, given a function $R(x, y) \operatorname{prop}_{s}[x \in \mathrm{N}, y \in A]$ the subset

$$
\alpha_{R} \equiv\left\{l \in A^{*} \mid \forall n \in \mathrm{N}\left(n<\mathrm{lh}(l) \rightarrow R\left(n, l_{n+1}\right)\right)\right\}
$$

where $\operatorname{lh}(l)$ is the length of $l$, turns out to be a formal point.

An alternative proof follows after noting, as observed in [Sig95], that the tree formal topology over a set $A$ is isomorphic to the exponential formal topology of the discrete formal topology of $N$ over 
the discrete formal topology on the set $A$ (see [Mai05a] for a predicative treatment of exponentiation). Therefore its formal points are in bijection with functions, because every function between discrete topologies is continuous. This explains why we denote the tree formal topology with the symbol $A^{N}$.

The realizability interpretation in [Mai12], showing that real numbers (both as Dedekind cuts or Cauchy sequences) do not form a set, also shows that choices sequences as formal points of Cantor or Baire formal topology do not form a set either and hence they only form a proper collection. Therefore predicatively we can only work with the point-free topologies of usual Cantor and Baire spaces.

The equivalence of each of our point-free topologies with the corresponding point-wise topology of their formal spaces as in definition 4.7, i.e. spatiality, is not generally valid in emTT. Indeed, spatiality of our tree formal topologies amounts to the well known principle of Bar Induction, as first observed in [FG82].

Def. 4.15 (Bar Induction in topological form) In emTT $+\triangleleft_{A^{N}}$ the principle of Bar Induction is the following statement: for any given set $A$

$$
(\mathrm{BI}(\mathrm{A})) \quad \forall l \in A^{*} \forall V \in \mathcal{P}\left(A^{*}\right) \quad\left(\forall \alpha \epsilon \operatorname{Pt}\left(\triangleleft_{A^{N}}\right) \quad(l \epsilon \alpha \rightarrow \alpha \gamma V) \rightarrow l \triangleleft_{A^{N}} V\right)
$$

where

$$
V \curlyvee W \equiv \exists a \in A(a \epsilon V \wedge a \epsilon W)
$$

expresses that two subsets $V, W$ of a set $A$ overlap (see [Sam03]).

The above formulation of $\mathrm{BI}(\mathrm{A})$ means that the topology put on formal points of the tree $A^{*}$, that are its choice sequences, coincides with the pointfree one. Hence, Bar Induction implies that we can reason topologically on choice sequences by induction on finite sequences, given that the pointfree topologies are inductively generated (see [Sam08, Sam14]).

The usual Fan theorem in [Tv88] is then an instance of Bar Induction (see [FG82, GS07]):

Def. 4.16 (Fan theorem) We call Fan theorem the formulation $\mathrm{BI}(\{0,1\})$ of $\mathrm{BI}(\mathrm{A})$ on Cantor formal topology, namely when $A \equiv\{0,1\}$.

Spatiality of Cantor formal topology allows to derive compactness of Cantor space [FG82].

In [Mai12] it is shown that emTT is compatible with the described principle of $\operatorname{Bar} \operatorname{Induction} \operatorname{BI}(A)$ for any set $A$, and the identification of lawlike sequences with recursive ones. Indeed, the realizability interpretation in [Mai12] showing that real numbers and choice sequences do not form a set validates the following principle called Formal Church thesis for operations between natural numbers

$$
\left(\mathrm{CT}_{\mathrm{tt}}\right)^{7} \quad \forall f \in \mathrm{N} \rightarrow \mathrm{N} \quad \exists e \in \mathrm{N} \quad \forall x \in \mathrm{N} \exists y \in \mathrm{N} \quad\left(T(e, x, y) \wedge U(y)=_{N} f(x)\right)
$$

stating that all operations between natural numbers are recursive.

Hence, the realizability interpretation in [Mai12] shows that emTT is compatible with constructive foundations where Bar Induction or Fan theorem is used, as in Brouwer's constructive pointwise approach of topology, by keeping a computable interpretation of operations between natural numbers with the validity of $\mathrm{CT}_{\mathrm{tt}}$. Actually a motivation to develop our minimalist foundation was exactly to study a development of topology in the presence of these extra axioms.

Observe that compatibility with Bar Induction and $\mathrm{CT}_{\mathrm{tt}}$ is not possible for Martin-Löf's type theory, because the axiom of choice, and hence also the axiom of unique choice, is valid in there. To see this, first observe that in our minimalist foundation we can prove the well known result by Kleene [Tv88] that Church thesis for choice sequences is contradictory with Fan Theorem, and hence also with Bar Induction. Observe then that this result can be formulated by saying that there is no model of emTT $+\triangleleft_{A^{N}}+\mathrm{FT}+\mathrm{CT}_{\mathrm{tt}}+\mathrm{AC} !_{\mathrm{N}, \mathrm{N}}$. Therefore a theory validating the axiom of unique choice cannot keep together a computational interpretation of operations and Bar Induction. Hence, as a consequence of the consistency of emTT with Bar Induction and $\mathrm{CT}_{\mathrm{tt}}$, it follows that the axiom of unique choice, and a fortiori the axiom of choice, is not valid in emTT.

\footnotetext{
${ }^{7}$ The subscript $t t$ stands for "type-theoretic functions", which we call operations in our foundation.
} 


\section{Concluding remarks and future work}

The existence of proper formal spaces, as the space of real numbers both as Dedekind cuts or Cauchy sequences, is in our opinion an advantage of a predicative and intuitionistic foundation as our minimalist one. In fact, a positive and practical motivation of the minimalist approach is to provide with a finer grid to look at reality, in particular topology, and thus preserve pieces of information, structures, conceptual distinctions (for example, all what is necessary to be able to instruct a computer) which would be lost, and actually are not even considered, in a classical or impredicative foundation. For instance, our minimalist foundation allows us to distinguish infinitary or ideal topological concepts not enjoying induction principles, like Brouwer's choice sequences, from inductive or real ones, as for example lawlike sequences. On the other hand, the minimalist attitude means that all results in the minimalist foundation about topology are valid also for the most relevant constructive and classical foundations.

Another important motivation supporting the pointfree approach to topology is given by a recent result showing that pointfree topology can be seen as a generalization of topology with points. To obtain this, one first has to introduce the notion of positive topology, that is an enrichment of formal topologies by the addition of a suitable primitive notion of closed subset. Then one can show that the category of concrete spaces can be embedded in the category of positive topologies. A full book [Sam14] on positive topologies and their developments is going to appear.

For the future, we plan to investigate topologies of real numbers and of choice sequences using the more powerful tool provided by positive topologies.

Acknowledgements: We heartily thank Francesco Ciraulo, Per Martin-Löf and Claudio Sacerdoti Coen for fruitful discussions on the topics of this paper.

\section{References}

[ABC ${ }^{+}$06] S. F. Allen, M. Bickford, R. L. Constable, R. Eaton, C. Kreitz, L. Lorigo, and E. Moran. Innovations in Computational Type Theory using Nuprl. Journal of Applied Logic, 4(4):428 469, 2006.

[Acz78] P. Aczel. The type theoretic interpretation of constructive set theory. In Logic Colloquium 'ry (Proc. Conf., Wroctaw, 1977), volume 96 of Stud. Logic Foundations Math., Amsterdam-New York, 1978. North-Holland.

[Acz82] P. Aczel. The type theoretic interpretation of constructive set theory: choice principles. In Dirk van Dalen Anne Troelstra, editor, The L.E.J. Brouwer Centenary Symposium (Noordwijkerhout, 1981), volume 110 of Stud. Logic Foundations Math., Amsterdam-New York, 1982. North-Holland.

[Acz86] P. Aczel. The type theoretic interpretation of constructive set theory: inductive definitions. In Logic, methodology and philosophy of science, VII (Salzburg, 1983), volume 114 of Stud. Logic Foundations Math., Amsterdam-New York, 1986. North-Holland.

[ARCT11] A. Asperti, W. Ricciotti, C. Sacerdoti Coen, and E. Tassi. The Matita interactive theorem prover. In Proceedings of the 23rd International Conference on Automated Deduction (CADE2011), Wroclaw, Poland, volume 6803 of LNCS, 2011.

[BB85] E. Bishop and D. S. Bridges. Constructive analysis. Springer, 1985.

[BCP03] G. Barthes, V. Capretta, and O. Pons. Setoids in type theory. J. Funct. Programming, 13(2):261-293, 2003. Special issue on "Logical frameworks and metalanguages".

[BDN09] A. Bove, P. Dybjer, and U. Norell. A brief overview of Agda - a functional language with dependent types. In S. Berghofer, T. Nipkow, C. Urban, and M. Wenzel, editors, Theorem Proving in Higher Order Logics, 22nd International Conference, TPHOLs 2009, volume 5674 of Lecture Notes in Computer Science, pages 73-78. Springer, August 2009.

[Bee85] M. Beeson. Foundations of Constructive Mathematics. Springer-Verlag, Berlin, 1985. 
[Bel88] J. L. Bell. Toposes and Local Set Theories: an introduction. Claredon Press, Oxford, 1988.

[Bis67] E. Bishop. Foundations of Constructive Analysis. McGraw-Hill Book Co., 1967.

[BR87] D. Bridges and F. Richman. Varieties of constructive mathematics., volume 97 of London Mathematical Society Lecture Note Series. Cambridge University Press, 1987.

[Bri08] D. Bridges. A reverse look at Brouwer's Fan Theorem. In One Hundred Years of Intuitionism (1907-2007), pages 316-325. Birkäuser, 2008.

[BS06] G. Battilotti and G. Sambin. Pretopologies and a uniform presentation of sup-lattices, quantales and frames. In Special Issue: Papers presented at the 2nd Workshop on Formal Topology (2WFTop 2002), volume 137 of Annals of Pure and Applied Logic, pages 30-61, (2006).

[CMS13] F. Ciraulo, M. E. Maietti, and G. Sambin. Convergence in formal topology: a unifying presentation. Logic and Analysis, 5(2):1-45, 2013.

[Coq90] T. Coquand. Metamathematical investigation of a calculus of constructions. In P. Odifreddi, editor, Logic in Computer Science, pages 91-122. Academic Press, 1990.

[Coq10] Coq development team. The Coq Proof Assistant Reference Manual: release 8.3. INRIA, Orsay, France, April 2010.

[CP90] Th. Coquand and C. Paulin-Mohring. Inductively defined types. In P. Martin-Löf and G. Mints, editors, Proceedings of the International Conference on Computer Logic (Colog '88), volume 417 of Lecture Notes in Computer Science, pages 50-66, Berlin, Germany, 1990. Springer.

[CSSV03] T. Coquand, G. Sambin, J. Smith, and S. Valentini. Inductively generated formal topologies. Annals of Pure and Applied Logic, 124(1-3):71-106, 2003.

[Cur10] G. Curi. On some peculiar aspects of the constructive theory of point-free spaces. Mathematical Logic Quarterly, 56(4):375-387, (2010).

[Fef79] S. Feferman. Constructive theories of functions and classes. In Logic Colloquium '78 (Mons, 1978), Stud. Logic Foundations Math., pages 159-224, Amsterdam-New York, 1979. NorthHolland.

[FG82] M. Fourman and R. J. Grayson. Formal spaces. In The L. E. J. Brouwer Centenary Symposium (Noordwijkerhout, 1981), volume 110 of Stud. Logic Foundations Math., pages 107-122. NorthHolland, 1982.

[GS07] N. Gambino and P. Schuster. Spatiality for formal topologies. Math. Structures Comput. Sci., 17(1):65-80, 2007.

[HJP80] J. M. E. Hyland, P. T. Johnstone, and A. M. Pitts. Tripos theory. Bull. Austral. Math. Soc., 88:205-232, 1980.

[Hof97] M. Hofmann. Extensional Constructs in Intensional Type Theory. Distinguished Dissertations. Springer, 1997.

[Hyl82] J. M. E. Hyland. The effective topos. In The L.E.J. Brouwer Centenary Symposium (Noordwijkerhout, 1981), volume 110 of Stud. Logic Foundations Math., pages 165-216. North-Holland, Amsterdam-New York, 1982.

[Ish05] H. Ishihara. Constructive reverse mathematics: compactness properties. In L. Crosilla and P. Schuster, editor, From Sets and Types to Topology and Analysis: Practicable Foundations for Constructive Mathematics, number 48 in Oxford Logic Guides, pages 245-267. Oxford University Press, 2005. 
[JM95] A. Joyal and I. Moerdijk. Algebraic set theory, volume 220 of Lecture Note Series. Cambridge University Press, 1995.

[Joh82] P. T. Johnstone. Stone Spaces. Cambridge U. P., 1982.

[JT84] A. Joyal and M. Tierney. An extension of the Galois theory of Grothendieck. Memoirs of the Amer. Math. Soc., 309, (1984).

[Mai98] M. E. Maietti. The type theory of categorical universes. PhD thesis, University of Padova, February 1998.

[Mai05a] M. E. Maietti. Predicative exponentiation of locally compact formal topologies over inductively generated ones. In From Sets and Types to Topology and Analysis: Practicable Foundation for Constructive Mathematics, volume 48 of Oxford Logic Guides, pages 202-222. Oxford University Press, 2005.

[Mai05b] M.E. Maietti. Modular correspondence between dependent type theories and categories including pretopoi and topoi. Mathematical Structures in Computer Science, 15(6):1089-1149, 2005 .

[Mai09] M. E. Maietti. A minimalist two-level foundation for constructive mathematics. Annals of Pure and Applied Logic, 160(3):319-354, 2009.

[Mai12] M. E. Maietti. Consistency of the minimalist foundation with Church thesis and Bar Induction. submitted (available via http://www.math.unipd.it/ maietti/pubb.html), 2012.

[Mar70] P. Martin-Löf. Notes on Constructive Mathematics. Almqvist \& Wiksell, 1970.

[Mar84] P. Martin-Löf. Intuitionistic Type Theory. Notes by G. Sambin of a series of lectures given in Padua, June 1980. Bibliopolis, Naples, 1984.

[Mar85] P. Martin-Löf. On the meanings of the logical constants and the justifications of the logical laws. In Proceedings of the conference on mathematical logic (Siena, 1983/1984), volume 2, pages 203-281, 1985. reprinted in: Nordic J. Philosophical Logic 1 (1996), no. 1, pages 11-60.

[MM92] S. MacLane and I. Moerdijk. Sheaves in Geometry and Logic. A first introduction to Topos theory. Springer Verlag, 1992.

[MR13] M. E. Maietti and G. Rosolini. Quotient completion for the foundation of constructive mathematics. Logica Universalis, pages 1-32, 2013. DOI 10.1007/s11787-013-0080-2.

[MRara] M. E. Maietti and G. Rosolini. Elementary quotient completion. Theory and Applications of Categories, Available via http://arxiv.org/pdf/1206.0162.pdf, to appear.

[MRarb] M. E. Maietti and G. Rosolini. Unifying exact completions. Applied Categorical Structures, Available via http://arxiv.org/pdf/1212.0966.pdf, to appear.

[MS05] M. E. Maietti and G. Sambin. Toward a minimalist foundation for constructive mathematics. In L. Crosilla and P. Schuster, editor, From Sets and Types to Topology and Analysis: Practicable Foundations for Constructive Mathematics, number 48 in Oxford Logic Guides, pages 91-114. Oxford University Press, 2005.

[NPS90] B. Nordström, K. Petersson, and J. Smith. Programming in Martin Löf's Type Theory. Clarendon Press, Oxford, 1990.

[NS99] S. Negri and D. Soravia. The continuum as a formal space. Archive for Mathematical Logic, 38(7):423-447, 1999.

[Pal05a] E. Palmgren. Bishop's set theory. Slides for lecture at the TYPES summer school, 2005. 
[Pal05b] E. Palmgren. Continuity on the real line and in formal spaces. In L. Crosilla and P. Schuster, editor, From Sets and Types to Topology and Analysis: Practicable Foundations for Constructive Mathematics, number 48 in Oxford Logic Guides, pages 165-175. Oxford University Press, 2005 .

[Rat05] M. Rathjen. Constructive set theory and brouwerian principles. J.UCS, 11(12):2008-2033, 2005.

[Sam87] G. Sambin. Intuitionistic formal spaces - a first communication. In D. Skordev, editor, Mathematical Logic and its Applications, pages 187-204. Plenum, 1987.

[Sam03] G. Sambin. Some points in formal topology. Theoretical Computer Science, 305:347-408, 2003.

[Sam08] G. Sambin. Two applications of dynamic constructivism: Brouwer's continuity principle and choice sequences in formal topology. In M. van Atten, P. Boldini, M. Bourdeau, and G. Heinzmann, editors, One Hundred Years of Intuitionism (1907-2007): The Cerisy Conference, pages 301-315. Birkhäuser, 2008.

[Sam11] G. Sambin. A minimalist foundation at work. In D. DeVidi, M. Hallett, and P. Clark, editors, Logic, Mathematics, Philosophy, Vintage Enthusiasms. Essays in Honour of John L. Bell, volume 75 of The Western Ontario Series in Philosophy of Science, pages 69-96. Springer, 2011.

[Sam12] G. Sambin. Real and ideal in constructive mathematics. In P. Dybjer, S. Lindström, E. Palmgren, and G. Sundholm, editors, Epistemology versus Ontology, Essays on the Philosophy and Foundations of Mathematics in honour of Per Martin-Löf, volume 27 of Logic, Epistemology and the Unity of Science, pages 69 - 85. Springer, NewYork/Dordrecht, 2012.

[Sam14] G. Sambin. The Basic Picture and Positive Topology. New structures for Constructive Mathematics. Oxford University Press, 2014. To appear.

[Sig95] I. Sigstam. Formal spaces and their effective presentations. Arch. Math. Logic, 34:211-246, 1995.

[SV98] G. Sambin and S. Valentini. Building up a toolbox for Martin-Löf's type theory: subset theory. In G. Sambin and J. Smith, editors, Twenty-five years of constructive type theory, Proceedings of a Congress held in Venice, October 1995, pages 221-244. Oxford U. P., 1998.

[Tv88] A. S. Troelstra and D. van Dalen. Constructivism in mathematics, an introduction, vol. I. In Studies in logic and the foundations of mathematics. North-Holland, 1988.

[Val07] S. Valentini. Constructive characterizations of bar subsets. Ann. Pure Appl. Logic, 145(3):368 $378,2007$.

[Wie06] F. Wiedijk. The Seventeen Provers of the World, volume 3600 of LNCS. Springer, 2006. 